\title{
Early complete response of primary bone marrow B-cell lymphoma treated with Rituximab-based CHOP therapy, assessed by flow cytometry and IGH rearrangement
}

\author{
Yoshimi Nabe ${ }^{1}$, Shohei Kikuchi ${ }^{1}$, Yusuke Kamihara ${ }^{1}$, Akinori Wada ${ }^{1}$, Jun Muarakami ${ }^{1}$, \\ and Tsutomu Sato ${ }^{1}$ \\ ${ }^{1}$ Toyama Daigaku Fuzoku Byoin
}

May 27, 2021

\begin{abstract}
Primary bone marrow B-cell lymphoma (PBML) is a special subtype of DLBCL which can be repeatedly sampled and evaluated by FCM and IGH rearrangement. Evaluation of early response by FCM and IGH assessments in the midpoint of treatment could be valuable for predicting treatment outcome.
\end{abstract}

Title: Early complete response of primary bone marrow B-cell lymphoma treated with Rituximab-based CHOP therapy, assessed by flow cytometry and IGH rearrangement

Authors: Yoshimi Nabe ${ }^{1,2}$, Shohei Kikuchi ${ }^{1}$, Yusuke Kamihara ${ }^{1}$, Akinori Wada ${ }^{1}$, Jun Murakami ${ }^{3}$, Tsutomu Sato $^{1}$,

${ }^{1}$ Department of Hematology, Toyama University School of Medicine, Toyama, Japan.

${ }^{2}$ Center for medical residency training, Toyama University School of Medicine, Toyama, Japan.

${ }^{3}$ Division of Transfusion Medicine and Cell Therapy, Toyama University School of Medicine, Toyama, Japan.

\section{Correspondence:}

Shohei Kikuchi M.D., Ph.D.,

Address: 2630 Sugitani, Toyama-city, Toyama 930-0152, Japan

E-mail: skikuchi@med.u-toyama.ac.jp

Telephone number: +81-76-434-7653 ext.7232

\section{Abstract}

Primary bone marrow B-cell lymphoma (PBML) is a special subtype of DLBCL which can be repeatedly sampled and evaluated by FCM and IGH rearrangement. Evaluation of early response by FCM and IGH assessments in the midpoint of treatment could be valuable for predicting treatment outcome.

\section{Key Clinical Message}

Evaluation of early response by FCM and IGH assessments against PBML could be valuable for predicting treatment outcome.

Keywords : Primary bone B cell lymphoma, IGH rearrangement, Rituximab-based CHOP 


\section{Introduction}

Primary bone marrow B-cell lymphoma (PBML) solely with large B-cell BM involvement at diagnosis is a rare form of aggressive B cell lymphoma, accounted for $1.23 \%$ of non-Hodgkin's lymphoma. ${ }^{1}$ PBML is considered as a distinct subtype of diffuse large B-cell lymphoma (DLBCL) with poor prognosis. ${ }^{1,2}$ The use of intensive induction therapies, such as R-Hyper CVAD or R-CHOP (rituximab, cyclophosphamide, doxorubicin, vincristine and prednisolone) followed by up-front high-dose chemotherapy and autologous stem cell rescue have been reported based on the assumption of poor prognosis. ${ }^{3,4}$ However, because of its rarity, the clinical features of PBML remain unclear and there is no evidence base to support a recommendation of intensive induction therapy.

\section{Case history,}

We report a 64-year-old man admitted with fever of several weeks' duration. Infection and collagen disease were excluded, but no diagnosis could be made. Computer tomography revealed no lymphadenopathy or extra-nodal tumor but 18-fluorodeoxyglucose-positron emission tomography (18 ${ }^{\mathrm{F}}$ FDG-PET) revealed increased uptake disseminated through the BM (SUV max 6.77, Figure 1A, B). Iliac crest BM biopsy showed large CD20+ B-cell infiltration, consistent with B-cell neoplasia (Figure 1C, D). Furthermore, flow cytometry (FCM) of BM aspirates showed increased CD19+ and CD20+ B-cell populations and marked kappa light chain restriction with $92.6 \%$ kappa and only $8.6 \%$ lambda was also observed in single color analysis (Figure 2A). Lymphoma cells expressing CD19 and surface immunoglobulin kappa were also increased with $74.5 \%$ (Figure 2B). Clonal immunoglobulin heavy chain (IGH) gene rearrangement was also detected by the polymerase chain reaction (PCR) (Figure 2C). Intravascular large B-cell lymphoma was suspected but excluded by random skin biopsy. Taken together, these data led to a diagnosis of PBML. R-CHOP therapy was initiated. Soon thereafter, fever completely resolved. Interim evaluation of BM aspirates after two cycles of R-CHOP showed a normal ratio of kappa to lambda with $18.2 \%$ to $17.0 \%$ (Figure 2A). IGH gene rearrangement abnormalities disappeared completely (Figure 2D), suggesting an early major response to R-CHOP therapy. After completion of six cycles of R-CHOP therapy, FDG-PET and BM examination were repeated, confirming a metabolic complete response (CR) (Figure 3A, B) with maintained normal kappa/lambda ratio, disappearance of CD19 and surface immunoglobulin kappa double positive lymphoma cells and lack of genetic abnormality (Figure 2A, B).

\section{Discussion,}

In this report, we have described a case of PBML with an early response confirmed by FCM and IGH gene rearrangement analysis. Thus, early chemosensitivity assessment could have predictive value for CR or $\mathrm{PFS}^{5,6}$ and avoid unnecessarily aggressive treatments. Genetic assays of IGH rearrangement have previously been employed for detecting minimal residual disease in B-cell leukemia. ${ }^{7}$ Unlike common DLBCL forming solid tumors, PBML is a special subtype of DLBCL which can be repeatedly sampled and evaluated by FCM and IGH rearrangement, as with leukemia. Early disappearance of IGH abnormalities could be a promising biomarker for major response to chemotherapy in PBML. Furthermore, repetitive evaluation for IGH abnormalities after treatment would be useful for guaranteed complete response or detection of early relapse.

\section{Conclusion}

PBML is a rare DLBCL entity with potentially poor prognosis but conventional R-CHOP therapy could be curative. To establish guidelines for standard therapies and to robustly predict prognosis, data on more cases are needed. Evaluation of early response by FCM and IGH assessments could be valuable for predicting treatment outcome and to optimize treatment intensity.

Authorship list and contribution: YN and SK collected data and drafted the manuscript. YN, SK, YK and AW were physicians providing chemotherapy. All authors reviewed the manuscript. TS supervised the study.

\section{Acknowledgments}


Financial support: none

Conflict of interest: The authors have no conflict of interest

Ethical approval: This article does not contain any studies with human participants performed by any of the authors.

Consent for publication: The written informed consent was obtained from the patient for publication.

\section{References}

1. Chang H, Hung YS, Lin TL, et al. Primary bone marrow diffuse large B cell lymphoma: a case series and review. Ann Hematol . 2011;90(7):791-796.

2. Martinez A, Ponzoni M, Agostinelli C, et al. Primary bone marrow lymphoma: an uncommon extranodal presentation of aggressive non-hodgkin lymphomas. Am J Surg Pathol . 2012;36(2):296-304.

3. Reed A, Sommerhalder D. The Use of R-Hyper-CVAD in a Rare Case of Primary Bone Marrow Diffuse Large B-Cell Lymphoma. J Hematol . 2019;8(4):165-167.

4. Hishizawa M, Okamoto K, Chonabayashi K, Kaneko H, Watanabe M, Tsudo M. Primary large B-cell lymphoma of the bone marrow. Br J Haematol . 2007;136(3):351.

5. Alvares CL, Matutes E, Scully MA, et al. Isolated bone marrow involvement in diffuse large B cell lymphoma: a report of three cases with review of morphological, immunophenotypic and cytogenetic findings.Leuk Lymphoma . 2004;45(4):769-775.

6. Haioun C, Itti E, Rahmouni A, et al. [18F]fluoro-2-deoxy-D-glucose positron emission tomography (FDG-PET) in aggressive lymphoma: an early prognostic tool for predicting patient outcome. Blood . 2005;106(4):1376-1381.

7. Wu D, Emerson RO, Sherwood A, et al. Detection of minimal residual disease in B lymphoblastic leukemia by high-throughput sequencing of IGH. Clin Cancer Res . 2014;20(17):4540-4548.

Figure Legend

\section{Figure 1}

Intense uptake of 18-fluorodeoxyglucose (FDG) disseminated throughout the bone marrow at initial diagnosis in the FDG-PET maximum intensity projection mode (MIP) (A). Uptake by the bone marrow at the sternum, vertebrae and pelvis (SUV max, 6.77; Deauville five-point scale: 4) (B). Histopathology of the bone marrow biopsy showing proliferation of large lymphoid cells; immunohistochemical staining showing HE (C) and CD20-staining (D) (magnification, x400).

\section{Figure 2}

Kappa/lambda ratio calculated by percentage of surface immunoglobulin kappa or lambda positive cells using flowcytometric analysis (A). Flowcytometric analysis. Lymphoma cells with CD19 and surface immunoglobulin kappa double positive at diagnosis and after 6 cycles of R-CHOP (B). IGH rearrangement analysis showing positive clonality peak at $\mathrm{VH}(\mathrm{FR} 1) / \mathrm{JH}$ region at the time of diagnosis $(\mathrm{C})$ and disappearance of clonality peak after two cycles of R-CHOP therapy (D).

\section{Figure 3}

FDG uptake by bone marrow after six-cycles of R-CHOP therapy by MIP imaging (A). Uptake by bone marrow of the sternum, vertebrae and pelvis, equal or lower than by the mediastinum (Deauville five-point scale: 2) (B). 
(A)
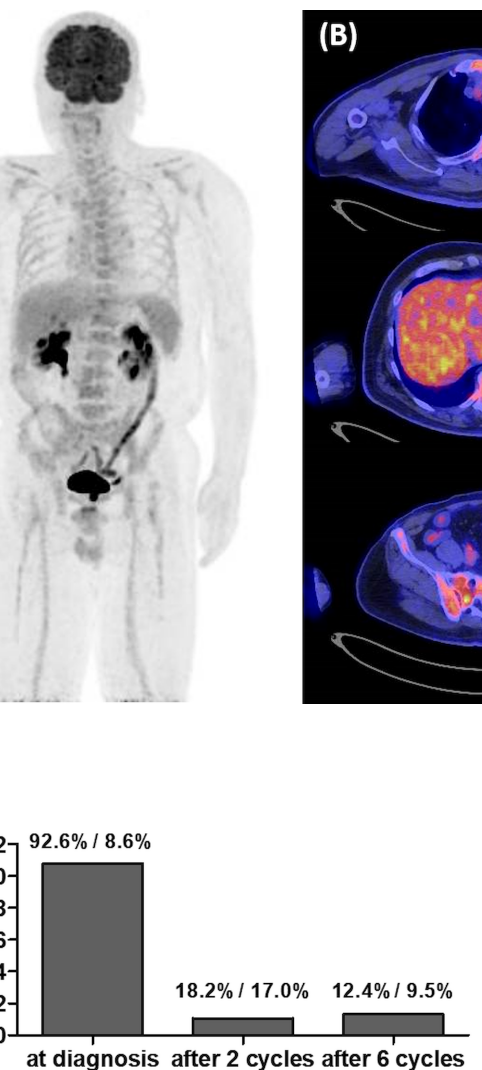

(A)

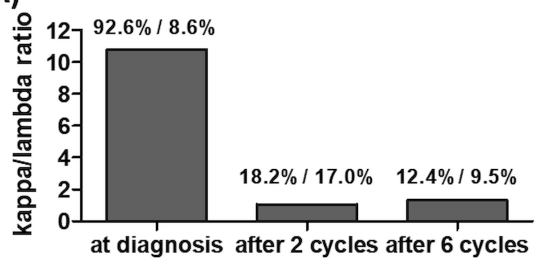

(B)

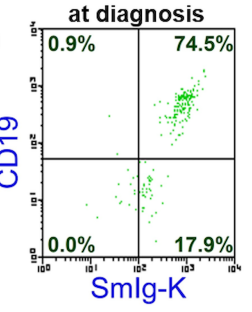

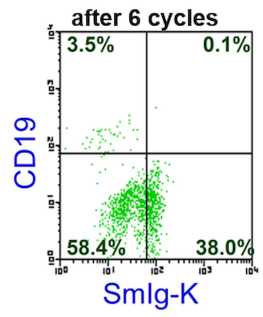

(C)

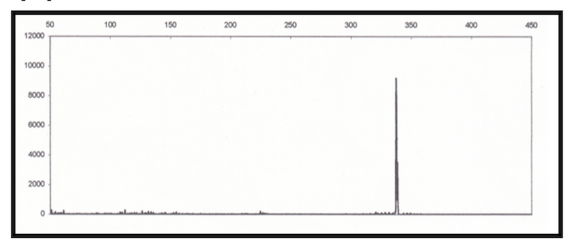

(D)

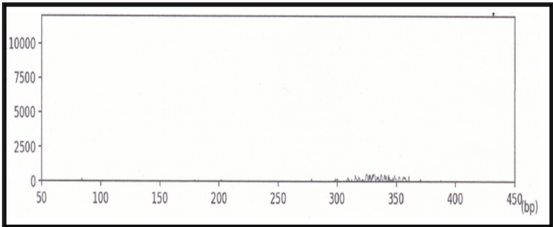




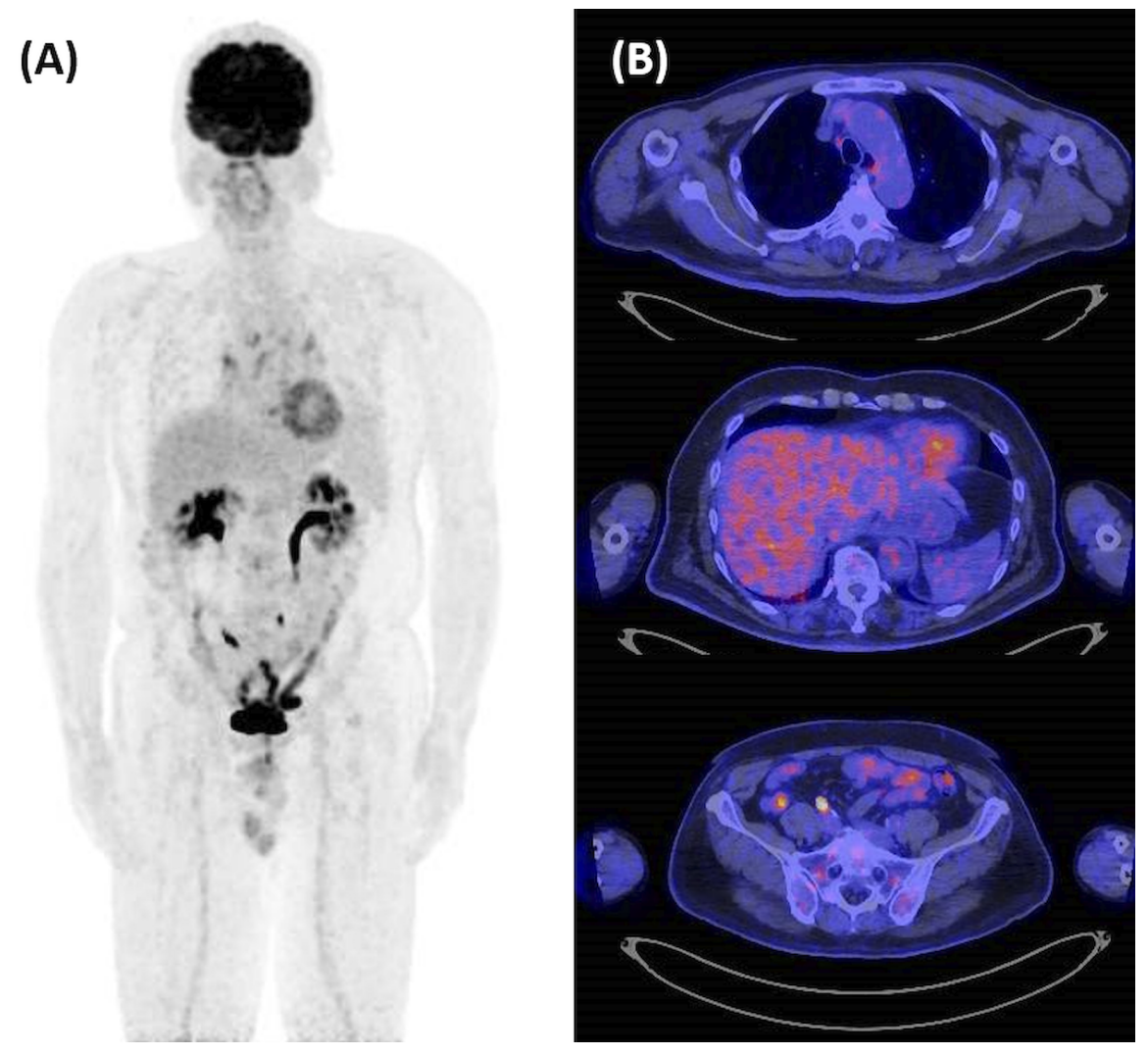

